\section{LES GOUVERNANTES-PHILOSOPHES: LA FEMME ENTRE FAMILLE ET ÉTAT DANS LA RÉPUBLIQUE DE PLATON ET DANS L'HYPER-TRADUCTION DE BADIOU}

THE RULERS-PHILOSOPHERS: WOMAN BETWEEN FAMILY AND STATE IN PLATO'S REPUBLIC AND IN BADIOU'S HYPER-TRNASLATION

Lorena Grigoletto Università degli studi di Napoli “Federico II"

\section{RESUME :}

Comment penser à l'égalité et à la différence How to think equality and gender gap in entre les genres au domaine politique ? Dans political arena? In Plato's Republic SocratesLa République de Platon Socrate-Badiou, Badiou, discussing with the female character dans son dialogue avec le personnage féminin Amantha, questions the consequences d'Amaranta, il critique les conséquences de of absolute equality among the class of cet égalité absolue lre les gouvernants - philosophers-rulers in Plato's originl text. In philosophes établie il y a plus de deux mille piscriticist the philosophes etablie ily a plus de deux mille hiscritism, the role of the woman is decisive, ans par le Socrate de Platon. De cette façon, la for it becomes the symbol of the necessary femme semble acquérir le papier symbolique difference between public and private sphere. d'articulation entre une dimension publique et privée.

KEY wORDS:

MOTS CLES :

Equality, State, family, rulers-philosophers.

Égalité, État, famille, gouvernantes, philosophes.
J'étais descendu hier au Pirée avec Glaucon, fils a d'Ariston, pour prier la déesse et voir, en même temps, de quelle manière on célébrerait la fête qui avait lieu pour la première fois. La pompe des habitants du lieu me parut belle, encore que moins distinguée fut celle que les Thraces conduisaient. Après avoir fait nos prières et vu la cérémonie, nous revenions vers la ville lorsque, nous ayant aperçus de loin sur le chemin du retour, Polémarque, fils de Céphale, ordonna à son petit esclave de couri après nous et de nous prier de l'attendre. L'enfant, tirant mon manteau par derrière: «Polémarque, dit-il, vous prie de l'attendre». (Platon: 1 )

Le jour où toute cette immense affaire commença, Socrate revenait du quartier du port, flanqué du plus jeune frère de Platon, un nommé Glauque. Ils avaient fait la bise à la déesse des Gens du Nord, ces marins avinés, et n'avaient rien manqué de la fête en son honneur, une grande première! Ç'avait de la gueule, du reste, le défilé des natifs du port. Et les chars des Gens du Nord, surchargés de dames très découvertes, n'étaient pas mal non plus.

Parmi les innombrables types nommés Polémarque, celui qui est le fils de Céphale les vit de loin, et lança un gamin à leurs trousses. «Attendez-nous!» vociféra le jeune gars en tirant la veste de Socrate. (Badiou, 2012: 1)

Circonstances similaires, mais deux destins différents; ou bien un seul, qui est celui d'une des œuvres les plus importantes de la pensée occidentale.

Composé entre les années Quatre-vingt et Soixante-dix du IV ${ }^{\circ}$ siècle A.J., la République est un dialogue célèbre, consacré au thème de la justice, qui présente Socrate engagé dans une discussion dans la maison de Céphale où il avait été invité par Polémarque.

Les personnages qui participent activement à la conversation sont Glauque et Adimante, les frères de Platon, et le sophiste politique Thrasymaque de Calcedonie. Les autres, Polémarque, Lisias, Euthydème, Nicerate, fils de Nicia, Charmantide de Paeanée et Clitofonte, fils d'Aristonyme, ne sont que des simples spectateurs et parfois ils proposent quelques répliques.

Ou, peut-être il y avait-t-il Socrate, sûrement, Amantha, la sœur de Platon, Glauque, Céphale, Polémarque, Thrasymaque et Clitofonte? Dans l'«hyper-traduction» ${ }^{1}$ (Boni, 2013: 411) de Alain Badiou, philosophe français contemporain naît à Rabat en 1937, dont $l^{\prime}$ «ultra-platonisme»(Boni, 2013: 412) montre une référence constante à la lecture lacanienne, les circonstances du dialogue changent significativement par rapport à la lecture traditionnelle. Socrate-Platon et Socrate-Badiou sont séparés par plus de deux mils ans de pensée philosophique qui, ne passant pas trop silencieusement, offrent parfois au protagoniste l'occasion de réfuter l'interprétation platonicien ou de la forcer.

1 Avec hyper-traduction on entend une traduction où la relation entre l'auteur et le traducteur est incliné en faveur de ce dernier. De cette façon, les intentions théoriques du traducteur remplacent en partie celles originales. 
Pourtant, l'introduction dans le dialogue d'un personnage féminin, à savoir Amantha (Adimante dans l'œuvre de Platon), confère à Badiou un regard plus large et renouvelé C'est justement cette fille, intransigeante et sarcastique, à introduire l'épineuse question de la participation des femmes à la vie politique contenue dans le Livre $\mathrm{V}$ et, en suite,à défendre la poésie de la nécessaire excommunication platonicienne dans le Livre $X$

Cependant, il est préalablement nécessaire de formuler théoriquement chacune des deux œuvres.

Comme il affirme à bon escient Giovanni Reale (Reale, 2009: 20), qui se réfère aux études de Eric A. Havelock et à son livre fondamental Preface to Plato de 1963, le titre République ne réfléchit pas le contenu de l'œuvre si l'on considère que seulement un tiers de cette dernière aborde des questions spécifiquement politiques, tandis que la plus large partie porte sur la "condition humaine" et, en particulier, sur l'éducation traditionnelle grecque dont il critique les principes.

En effet, le caractère révolutionnaire de l'œuvre est dû surtout à l'objectif de bouleverser la paideia grecque, c'est-à-dire la structure propre à la culture de l'oralité poétique-mimétique; une véritable révolution qui concerne le langage dont but est théorique-morale. La culture de l'homme grec se bornait à l'exercice mnémonique et répétitif complètement identifié aux modèles «polyvalents» offerts par les poètes. Or, à partir dePlaton,l'hellène auraitvusonéducationinspiréeà une mentalité philosophiqueconceptuelle, soit à un modèle nouveau et univoque orienté vers la connaissance du Bien. Mnémosyne, mère de toutes les muses, fondamental dans la formation de l'homme grec, elle assumait donc une nouvelle signification et une nouvelle valeur. À l'éducation traditionnelle,fondésurlapoésie,onremplaçaitunmodèleéducatifbasésurlaphilosophie. Le grand objectif critique de Platon est donc la poésie. Or, il ne s'agit pas de la poésie en général, qui sera pensée à nouveau en relation aux axiomes de l'État, mais seulement à l'epos omérique-esiodé qui, par le biais de l'imitation (mimesis), pousse à l'identification émotive, soit à la perte d'objectivité dans des modèles qui conduisent l'âme vers sa partie passionnelle.

Pourtant, nous sommes censés faire une clarification importante: la question politique ne montre son sens que par son intime connexion avec les principes théoriques; l'État platonicien constitue la voie d'accès au Bien, qui est le véritable fondement théologique, dans la communauté des hommes par les philosophes qui ont su s'élever. C'est grâce à ce Principe, fondement de l'Être, du cosmos, de l'âme et de la dimension publique de la vie de l'homme, que, la tripartition de l'âme en faculté rationnelle, passionnelle et concupiscible, correspond par analogie à la tripartition de l'État dans les classes suivantes: Artisans, Guerriers et Gardiens. Ainsi, aux vertus de l'État correspondent les vertus du citoyen.
Donc, à la question inaugural du dialogue «Qu'est-ce que c'est la Justice?», à laquelle Thrasymaque répond en soutenant qu'elle est l'intérêt du plus fort, Socrate-Platon répond en offrant une définition convenable tant à l' État qu'à l'individu. La Justice est la vertus (areté) qui consiste à faire en sorte que chaque partie exerce sa fonction, en introduisant de l'ordre dans le désordre, et de l'unité dans la multiplicité; cela explique la santé ou la maladie du corps métaphoriquement entendu.

Alain Badiou, penseur militant, se propose des objectifs différents. En effet, il veut indiquer l'hypothèse communiste, relative à la classe des Guerriers et des Gardiens, comme étant enracinée dans le dialogue entre la philosophie et la politique, en individuant dans le platonisme un fondement philosophique. Au même temps, tout en se tenant à une perspective contemporaine, la structure unitaire de l'œuvre platonicienne lui sert à montrer les directives de sa réflexion en défense d'une pensée «forte», systématique et liée aux catégories classiques de la métaphysique. Et encore: le thème de la relation entre philosophie et poésie apparaît névralgique au point d'évoquer la spéculation heideggerienne qui fait de la poésie la source de la pensée.

Donc, la critique platonicienne à la mimesis donne à Badiou l'occasion de revendiquer sa thèse d'inésthétisation de l'opération poétique qui, par le biais d'une «méthode soustractive» refuse la façon esthétique et présente les opérations rationnelles de la poésie en lui restituant sa dimension spéculative.

Dans son interprétation, ce qui change est aussi bien la terminologie: l'individu est remplacé par le Sujet. En effet, chez Platon l'acteur rationnel (l'homme) n'avait pas d'autres significations franchissant la collectivité, bien que l'introduction du concept d'âme immortelle (psyché athanatos) a fait qu'elles étaient déjà beaucoupplus développées par rapport à la culture pré-hellénistique. Donc, l'analogie entrela tripartition de l'âme et celle de l'État est bien plus qu'une simple correspondance qui trouve dans la Justice sa valeur structurelle. En effet, cet écart entre l'individu platonicien, qui est impossible à penser en dehors de la collectivité, et le Sujet de Badiou explique le choix du philosophe français de distinguer la «justice objective» de la «justice subjective». Cette dernière étant pensée en termes de hiérarchie (être «musicien de soi même») parmi les instances constitutives du Sujet (Pensée, Affect, Désir) qui remplacent la traditionnelle tripartition du philosophe grec.

Pour sa part, la «justice objective», c'est-à-dire celle qui se réfère à la collectivité, montre un caractère inédit. En effet, à la capacité d'accomplir sa propre fonction, c'est à dire de suivre le chemin que l'on considère le plus conforme à sa propre nature, il s'ajoute l'habilité transversale qui, suivant la terminologie marxiste, est celle du «travailleur polymorphe» ayant la capacité d'accomplir n'importe quel rôle ou fonction sociale; «un animal humain qui [...] ne laisse en dehors de son champ d'action aucune des possibilités que l'époque lui propose» (Badiou, 2013: 157). 
La «Justice objective» se configure ainsi comme le lien dialectique de «localisation» et $\mathrm{d}^{\prime}$ «ouverture» entre les dispositions subjectives et leur vitalité sociale. À ce propos, Amantha souligne que tant la disponibilité pour toute praxis que le développement de l'exis de chacun sont nécessaires afin d'empêcher les horreurs produits autant par un excès de homogénéité que par son défaut total. Ainsi, l'opportunité d'affirmer $\mathrm{l}^{\prime}$ «injustice selon l'égalité» et $\mathrm{l}^{\prime}$ «injustice selon la liberté», s'ouvre au lecteur de la République.

Donc, $l^{\prime}$ «homme polyvalent» de la culture grecque, à laquelle Platon dirige sa critique, se retrouve ici noué avec le «travailleur polymorphe» de Marx, tout en soulignant l'incommensurabilité entre les deux positions, c'est-à-dire l'écart entre la dimension étique-théorique de Platon et celle politique de Badiou et, de pair, entre l'articulation de l'homme grec dans la collectivité et celle de Sujet dans la culture moderne.

Évidemment Badiou, en interprétant l'État platonicien en termes de communisme ou bien, suivant sa définition, en terme $\mathrm{d}^{\prime}$ «aristocratie populaire», il le rapproche au «collectivisme moderne»; il faut rappeler, pourtant, les différences historiques et théoriques. Ce dernier, en effet, présuppose la révolution industrielle, le capitalisme et la centralité de la classe travailleuse, au contraire, «le motivazioni teoretiche di questo comunismo (celui platonicien) sono decisamente spiritualistiche e quasi ascetiche» (Reale, 2009: 61).

Mais qu' est-ce que c'est concrètement le «communisme»? Qu'est-ce que c'est ce que l'on partage? C'est-à-dire qu'est-ce que c'est qui produit Koinonia (communauté) entre les membres de la politeia? Qu'est-ce que c'est ce qui produit l'harmonie oula santé dans le corps social? Platon nous répond dans le Livre $\mathrm{V}$ de la République,où il montre comme la véritable unité psycho-politique-physiologique est possible seulement à partir de l'unité de la classe politique qui la conquiert par le biais de la vie communautaire. Une telle unité ne peut exister que si l'on partage autant le plaisir que la douleur, c'est-à-dire si l'on partage la première structure sociale, la famille et donc les femmes et les enfants.

Ainsi, le Livre $\mathrm{V}$, qui constitue la première tentative de reconnaître à l'univers féminin le droit de "citoyenneté", en s'opposant à la culture dominante qui lui réserve un rôle de subordination dont Aristote est l'autorité majeure, s'inscrit dans un cadre théorique qui montre les raisons profondes.

Et moi j'allais les dénombrer dans l'ordre où elles me paraissaient se former les unes des autres, lorsque Polémarque, qui était assis un peu plus loin qu'Adimante, avançant la main saisit ce dernier à l'épaule par son manteau, le tira à lui et, se penchant, lui dit ̀̀ voix basse quelques mots dont nous n'entendîmes qu lui et, se penchant, lui dit à voix basse quelques mots dont nous n'endînes que ceux-ci, "Ĺpe laissendit Adinous ṕsser outre ou que ferons-nous?» "Pas le moins du monde», répondit Adimante, élevant déjà la voix. «Qu'est-ce au juste que vous ne voulez
pas laisser passer?» «Toi», dit-il. «Et pour quelle raison?» «Il nous semble que tu perds courage, que tu nous dérobes toute une partie, et non la moindre, du sujet pour n'avoir pas à l'étudier, et que tu t'imagines nous échapper en disant à la légère qu'à l'égard des femmes et des enfants tout le monde trouverait évident qu'il y eût communauté entre amis». «Ne l'ai-je pas dit à bon droit, Adimante?» «Si», concéda-t-il; «mais ce bon droit-là, comme le reste, a besoin d'explications. Quel sera le caractère de cette communauté? Il y en a, en effet, beaucoup de possibles». (Platon: 161)

Socrate s'apprête à énumérer, dans l'ordre logique et historique qui règle leur interdépendance, les quatre politiques insuffisantes, quand Polémarque touche l'épaule nue d'Amantha. La dure jeune fille se cabre, puis comprend que son voisin ne veut qu'attirer son attention. Elle se rapproche et lui murmure à l'oreille:

«Allons-nous le laisser franchir l'obstacle comme s'il ne l'avait pas même vu?» «Il faut absolument l'en empêcher, rétorque Amantha. «Qui faut-il empêcher de faire quoi?» se retourne Socrate. «Vous, dit Amantha, de nous prendre pour des benêts!» «Diable! Qu' ai-je donc fait?» «Vous nous traitez avec une impardonnable légèreté, permettez-moi de vous le dire, répond une Amantha, très remontée. Vous passez par-dessus une question de la plus haute importance uniquement pour nous passez par-dessus une question de la plus haute inpoir pas a vous mouiller. Croyez-vous ça au détour de la conversation, que, s'agissant des femmes et des enfants, il est évident - je cite votre formule - "qu'entre amis on partage tout" " «Mais, chère Amantha, n'est-ce pas vrai?» «à moins qu'il ne s'agisse d'une cochonnerie, moi, en tant que jeune femme, je ne sais même pas ce que cette phrase veut dire: que s'agit-il au juste de "partager"?» (Badiou, 2013: 179)

L'argumentation platonicienne et, évidemment, celle badousiénne, très difficile à expliquer, est celle de la différence entre les genres qui légitime la participation des femmes au pouvoir politique. La différence entre la femme et l'homme n'est pas absolue mais relative au domaine auquel on fait référence, ce qui revient à dire que, sur le plan politique, on ne peut pas dire que la femme a moins de capacités par rapport à l'homme.

Le Socrate de Badiou réfléchit sur le fait que, comme la différence entre un chauve et un chevelu acquiert une certaine importance en référence à l'être client d'un coiffeur, le même écart s'avère être sans valeur quand il est rapporté à la pêche à la ligne. Ainsi, la différence entre les genres est radicale si l'on pense au processus de reproduction mais elle n'est pas si importante dans le domaine politique.

En effet, chacun poursuit ses inclinations spécifiques tant les femmes que les hommes, bien que les premières elles aient mois de force; la différence n'est donc pas selon la qualité mais selon la quantité. Comme parmi les chiens les femelles et les mâles accomplissent les mêmes fonctions de conduction du troupeau, à la même manière, dans le gouvernement de l'État, il n'y a pas des rôles exclusifs d' aucun genre.

Comme on peut bien l'observer il y a des femmes qui sont inclines naturellement à des activités particulières comme la cuisine, la musique, mais aussi à la guerre et 
à la gouvernance. Selon Socrate-Platon, donc, les femmes devront recevoir la même éducation que les hommes.

Il est absurde, cependant, comme Amantha le souligne, de soutenir la nécessité que l'État soit géré selon sa nature unique (selon l'unité de son Principe), en s'appuyant sur la compétence universelle qui nous a indiqué la définition de «justice objective», sans considérer la différence objective et subjective entre les genres; une différence que la jeune disciple définit ontologique et «symbolique». À qu'est-ce qu'elle pense Amantha? Peut-être aussi à une relation différente parmi les trois instances du Sujet (Pensée, Affect, Désir)? La différence symbolique entre les genres, qui se présente dans le débat philosophique contemporain en termes d'opposition entre société du Père² et «ordre symbolique de la mère» (Muraro, 2006), est-ce qu'il montre peut-être la difficulté d'articuler l'unité du Principe, c'est-à-dire l'unité de la Pensée, avec les positions d'une «pensée de la différence»? De toute façon, Badiou défend l'égalité politique entre les genres avec des considérations actuelles; en effet, l'apparente inadéquation des femmes au gouvernement est le produit de «brutales ségrégations éducatives et d'insidieuses propagandes sur la prétendue "faiblesse" du sexe féminin» (Badiou, 2013: 188)

$\mathrm{Au}$ contraire, Platon ne poursuit pas l'objectif de donner de la valeur à l'univers féminin, mais il suit une visée philosophique de constituer le corps communautaire, soit l'unité de l'État en vertu du Principe: le Bien. En effet, on peut pas qualifier les locutions comme «avoir en commun femmes et enfants» (Platon), ou «entre amis on partage tout» (Badiou) comme des expressions philogines.

Or, c'est dans ce contexte qui s'inscrit le véritable problème: il est possible d'acquérir cette unité seulement si l'on efface la structure familiale qui, par le biais des liens parentales, produit des liens patrimoniales. Donc, ce n'est que grâce à son abolition que les classes des Guerriers et des Gardians pourront se consacrer in toto à gouverner et à protéger l'État en puisent à la source du Bien. Dans la classe des Artisans, par contre, cela ne sera pas nécessaire. La famille particulière, chez les premiers, sera substituée par une famille plus grande où les liens parentales seront étendus à l'ensemble du corps politique, où partager les plaisirs et les douleurs signifie d'empêcher la dissolution de l'unité du Bien. Exclusion faite du corps de chacun, toute chose sera partagée. En effet, sans la propriété privée il est possible de rejoindre une coïncidence totale entre la dimension publique et la dimension privée, c'est-à-dire entre le Bien et les biens particuliers.

Dans la réflexion de Badiou, la famille est définie en termes de «pilier de l'ordre inégalitaire» ou bien comme le «fardeau réactionnaire», car elle est le lieu où se

2 Dans Vers la société sans pères (1963) Alexander Mitscherlich, médecin et psychanalyste allemand, nous montre le processus, commencé dans les années Soixante sous la pression politique anti-autoritaire, d'élimination progressive du père comme principe hiérarchique, mais aussi comme modèle de l'autorité identifiée par connotations symboliques et affectives. concentre la richesse; de fait, elle naît avec la propriété privée. Engels, L'Origine de la famille, de la propriété privée et de l'état, devient alors une référence obligatoire, selon la jeune Amantha qui en soutient les principes.

Cependant, comme il nous explique en première instance le Socrate de Platon, il s'agit d'une condition possible à conquérir seulement par l'intervention de l'État en effet, l'État substituera ce que Badiou appelle «l'ordre symbolique de la filiation» (Badiou, 2013: 190), avec un projet idéologique et, finalement, eugénique, qui décidera les accouplements avec des faux tirages.

Et pendant que Glauque, le disciple de Socrate-Badiou, pose la question éternelle «Qui va s'occuper des enfants?», (c'est-à-dire comment la gouvernante-philosophe pourra-t-elle partager son temps entre la famille et son droit-devoir de "citoyenne"?), en affirmant la centralité de la femme dans le processus éducatif, Amantha, le personnage féminin de Badiou, croit à la nécessité d'abolir la structure familiale une fois que l'on assume l'exercice polyvalent des fonctions publiques. L'égalité politique entre les genres, absolument nécessaire pour obtenir l'égalité sociale des classes des Guerriers et des Gardians, il ne semble pourtant pas considérer la différence biologique et sociale entre la femme et l'homme. La question provocatrice que Glauque pose à SocrateBadiou, acquiert alors une certaine importance: «Accessoirement, que devient, dans ce contexte, l'égalité entre les femmes, qui portent l'enfant à naître dans leur ventre, qui nourrissent les fœetus avec leur propre sang, qui accouchent dans la douleur, qui allaitent le bébé, et les hommes qui n'ont rien fait dans cette histoire qu baiser et jouir?» (Badiou, 2013: 190)

Ainsi, l'interprétation de Badiou semble reconnaître dans la femme l'origine de la famille en tant que structure éducative, sociale et économique, c'est à dire le lieu où on peut affirmer pour la première fois " $c$ 'est à moi", mais aussi où il faut nécessairement le dire en vertu du rapport viscérale entre mère et enfant. En effet, $d$ ' après Socrate-Platon, la première chose à faire pour abolir cette structure sociale primaire, en permettant la cö̈ncidence entre privé et publique, c'est d'empêcher aux femmes d'exercer leur fonction naturelle de mères; ainsi, le droit de citoyenneté est acquis en dépit de leur nature, c'est-à-dire de leur rôle psycho-biologique qui implique l'inégalité sociale entre les genres et, par conséquence, l'inégalité politique. En tout cas, il faut rappeler que dans l'antiquité classique, le rôle éducatif était assumé entièrement par l'homme qui, étant le seul parent, considéré plus tard comme le Père créateur, représentait l'autorité éthique par excellence. Afin de réaliser ce projet donc, les meilleurs enfants seront donnés à des allaitantes pour qu'ils ne puissent plus reconnaître leurs familles. Pourtant, avec Badiou, $l^{\prime}$ «antifamilisme platonicien» a l'occasion de se soustraire à la définition du philosophe français qui décrit ses caractères principaux comme les horreurs de la Cité 
et, parallèlement, Socrate-Badiou peut se rebeller contre Socrate-Platon, à savoir contre son auteur original.

Votre frère aîné, Platon, a cru pouvoir parler en mon nom sur cet étrange et presque intraitable sujet qu' est la famille. [...] Et d' après le Socrate de ton frère, dans la trop fameuse "Cité idéale", on abolit la propriété privée et la famille, mais l'état sort de ces abolitions doté de pouvoirs exorbitants. A partir de l'axiome selon lequel les enfants appartiennent à la communauté toute entière on en vient, dans la ligne enfants appartiennent a la communaute toute entière, on en vient, dans la ligne de cet antifamilisme platonicien, à ce qu'il faut bien appeler des horreurs. [...] Chers amis, moi, Socrate, je ne paierai pas ce prix pour la nécessaire dissolution de a famille telle qu'elle est. No et non. Profitant de l'occasion qui m'en est donnée par Badiou, je m'élève ici solennellement contre l'interprétation de ma pensée par votre frère Platon. (Badiou, 2013: 191, 192)

«L'étatisation presque intégral des liens privés et la disparition de l'intime» (Badiou, 2013: 190) apparaissent au Socrate de Badiou non seulement comme une monstruosité mais aussi comme ce qui ne peut pas être réalisé, parce que l'amour, comme toute vérité non politique, nécessite d'un espace d'invisibilité. L'élimination de cet espace, soit de cet écart entre le publique et le privé n'est pas un péril lié uniquement à l'hypothèse communiste, mais aussi, aux démocraties corrompues, où les politiques racontent leurs relations extraconjugales et leurs activités sexuelles les plus bizarres.

Ainsi, Socrate-Badiou se distingue du Socrate-Platon et il semble accorder à la femme la valeur symbolique d'articulation entre la dimension publique et celle privée mais, de cette manière, il laisse entendre que l'inégalité s'insinue à nouveau dans l'État: si on ne peut pas faire abstraction de l'institution familiale, qui est nécessaire pour éviter les horreurs de la Cité, il en découle qu'aussi l'égalité sociale absolue ne peut pas être conservée.

L'écart entre Socrate-Platon et Socrate-Badiou, dû à plus de deux mils ans de pensée philosophique et à l'apparition d'une femme dans le dialogue, offre la possibilité de réfléchir sur un thème extrêmement contemporain. L'égalité politique entre les genres, obtenue par l'abolition de la famille, implique l'égalité sociale car elle efface le germe de la propriété privée, c'est-à-dire de l'inégalité, qui naît dans la famille; cependant, affirmer cette égalité politique et sociale signifie opter pour l'inégalité entre les genres, c'est-à-dire qu'on renonce à protéger leur différence. Qu'est-ce qu' on entend alors pour égalité et différence à niveau politique? En effet, si l'on pense au débat contemporain les adversaires des lois politiques vouées à protection des femmes, telle que les quotas, critiquent, tout d'abord, le présupposé sur lequel ces lois s'appuient: en effet, ils y voient à l'œuvre l'admission implicite de l'inégalité, en vertu de laquelle les femmes seraient considérées comme des sujets politiques faibles qu'il faut protéger. Deuxièmement, ils contestent l'inefficacité d'une telle décision politique qui ne serait soutenue par aucune réalité socio-culturelle. Eva Cantarella, l'une des principales spécialistes de droit antique, qui avec L'ambiguo malanno nous a bien informés sur la condition féminin aux temps anciens, ne s'exprime pas en faveur des quotas, en critiquant auss les opérations de «genrisation» de la production artistique et intellectuelle de l'univers féminin (Cantarella, 2012)

Est-ce que parler de «différence de genre» signifie reconnaître aussitôt le droit de l'un d'entre eux à ne pas se consacrer entièrement à l'activité politique? Est-ce que l'on peut parler encore de «égalité», absolue ou relative?

La position de Badiou et l'actualité de l'œuvre platonicienne nous obligent à reconsidérer le concept d'égalité et à en renouveler la valeur. Nous arrivons doncà hésiter sur la possibilité que derrière une déclaration d'égalité se cache une discrimination importante qui refuse ce qu'aujourd'hui on nommerait "le droit à la différence".

\section{BIBLIOGRAFIE}

Badiou, A., La République de Platon, Paris, Fayard, 2012.

Boni, L., Nota introduttiva «Platone, firmato Badiou», in La Repubblica di Platone (Badiou, A.), Milano, Ponte alle Grazie, 2013, pp. 5-9.

Boni, L., Postfazione, «Per un approfondimento critico: l'ultraplatonismo polimorfo di Alain Badiou», in La Repubblica di Platone (Badiou, A.), Milano, Ponte alle Grazie, 2013, pp. 411 418.

Cantarella, Eva, «Quote rosa e cultura. Intervista a Eva Cantarella sulle donne all'univerità». Meno di zero. Rivista dell'Università in Movimento. Internet. 15-12-2014. http://menodizero eu/passatopresente-analisi/264-quote-rosa-e-cultura-intervista-a-eva-cantarella-sulledonne-alluniversita.html

Giovanni, R., «Saggio integrativo», in Repubblica (Platone), Milano, Bompiani, 2009, pp. 624669

Giovanni, R., «Saggio introduttivo», in Repubblica (Platone), Milano, Bompiani, 2009, pp. 20127.

Mitscherlich, A., Verso una società senza padre. Feltrinelli, Milano, 1970

Muraro, L., L'ordine simbolico della madre, Roma, Editori Riuniti, 2006.

Platon, CEuvres complètes. La République, Paris, Librairie Garnier Frères, 1963. 